\title{
PILOT SCHEME FOR THE ESTABLISHMENT OF A NEONATAL SCREENING PROGRAM IN THE DEMOCRATIC REPUBLIC OF LAOS
}

\author{
T. Hoehn ${ }^{1}$, Z. Lukacs ${ }^{2}$, S. Bounnack ${ }^{3}$ \\ ${ }^{I}$ Neonatology, University Children's Hospital Duesseldorf, Duesseldorf, ${ }^{2}$ Neonatal Screening Laboratory, \\ Hamburg University Medical Center, Hamburg, Germany, ${ }^{3}$ Neonatology and Pediatrics, Mother \& Child \\ Hospital, Vientiane, Laos
}

Background: Neonatal screening programs have been established and are in use in many countries worldwide. Countries without any established neonatal screening programs in 2009 included Nepal, Cambodia, Laos, and the Pacific Island nations.

\begin{abstract}
Aim: To show the feasibility of neonatal screening in the urban setting of Vientiane, capital of Laos. Furthermore to expand regular screening to other cities within Laos and to offer ongoing teaching to local pediatricians with respect to further follow-up of infants with diagnosed inborn errors of metabolism.
\end{abstract}

Methods: Initial participants were the large maternity hospitals within the city of Vientiane (Mahosot Hospital [2.500 deliveries p.a.], Sethathirath Hospital [2.000 deliveries p.a.], Friendship Hospital [700 deliveries p.a.], and Mother \& Child Health Hospital [3.500 deliveries p.a.]). Samples were taken immediately prior to hospital discharge and once weekly air-shipped to a German screening laboratory.

Results: Currently approximately 11.440 samples of newborn infants have been examined. The rate of retests was above European average due to very early discharge policies in Laotian maternity hospitals. Confirmed cases of neonatal congenital diseases include two infants with hypothyroidism and one infant with congenital adrenal hyperplasia without salt-loss. All three infant received early therapy and are currently doing well.

Conclusions: Even in a very low resource setting as in Laos the establishment of a neonatal screening program appears to be feasible. Further challenges include the expansion to the rest of the country and the on-site establishment of measurements within Laos. 